University of Nebraska - Lincoln

DigitalCommons@University of Nebraska - Lincoln

\title{
9-1998
}

\section{Wavelength Conversion in WDM Networking}

\author{
Byrav Ramamurthy \\ University of Nebraska-Lincoln, bramamurthy2@unl.edu \\ Biswanath Mukherjee \\ IEEE
}

Follow this and additional works at: https://digitalcommons.unl.edu/csearticles

Part of the Computer Sciences Commons

Ramamurthy, Byrav and Mukherjee, Biswanath, "Wavelength Conversion in WDM Networking" (1998). CSE Journal Articles. 68.

https://digitalcommons.unl.edu/csearticles/68

This Article is brought to you for free and open access by the Computer Science and Engineering, Department of at DigitalCommons@University of Nebraska - Lincoln. It has been accepted for inclusion in CSE Journal Articles by an authorized administrator of DigitalCommons@University of Nebraska - Lincoln. 


\title{
Wavelength Conversion in WDM Networking
}

\author{
Byrav Ramamurthy, Student Member, IEEE, and Biswanath Mukherjee, Member, IEEE
}

\begin{abstract}
Wavelength conversion has been proposed for use in wavelength-division multiplexed networks to improve efficiency. This study highlights systems challenges and performance issues which need to be addressed in order to incorporate wavelength conversion effectively. A review/survey of the enabling technologies, design methods, and analytical models used in wavelengthconvertible networks is provided.
\end{abstract}

Index Terms - Circuit switching, lightpath, network design, optical network, performance analysis, wavelength conversion, wavelength-division multiplexing (WDM), wavelength routing.

\section{INTRODUCTION}

W AVELENGTH-DIVISION multiplexing (WDM) [1] is a promising technique to utilize the enormous bandwidth of the optical fiber. Multiple wavelength-division multiplexed channels can be operated on a single fiber simultaneously; however, a fundamental requirement in fiber-optic communication is that these channels operate at different wavelengths. These channels can be independently modulated to accommodate dissimilar data formats, including some ana$\log$ and some digital, within certain limits. Thus, WDM utilizes the huge bandwidth $(\sim 50 \mathrm{THz})$ of a single-mode optical fiber while providing channels whose bandwidths $(1-10 \mathrm{~Gb} / \mathrm{s})$ are compatible with current electronic processing speeds.

In a WDM network, it is possible to route data to their respective destinations based on their wavelengths. The use of wavelengths to route data is referred to as wavelength routing, and a network which employs this technique is known as a wavelength-routed network [2]. Such a network consists of wavelength-routing switches (or routing nodes) which are interconnected by optical fibers. Some routing nodes (referred to as crossconnects) are attached to access stations where data from several end-users could be multiplexed on to a single WDM channel. An access station also provides optical-toelectronic $(\mathrm{O} / \mathrm{E})$ conversion and vice versa to interface the optical network with conventional electronic equipment. A wavelength-routed network which carries data from one access station to another without any intermediate $\mathrm{O} / \mathrm{E}$ conversion is referred to as an all-optical wavelength-routed network. Such all-optical wavelength-routed networks have been proposed for building large wide-area networks [3].

Manuscript received July 1, 1997; revised April 2, 1998. This work was supported in part by DARPA Contracts DABT63-92-C-0031 and DAAH0495-1-0487; NSF Grants NCR-9205755, NCR-9508239, and ECS-9521249; Pacific Bell; and the UC MICRO Program.

B. Ramamurthy is with the Department of Computer Science and Engineering, University of Nebraska-Lincoln, Lincoln, NE 68588-0115 USA (e-mail: byrav@cse.unl.edu).

B. Mukherjee is with the Department of Computer Science, University of California, Davis, CA 95616 USA (e-mail: mukherjee@cs.ucdavis.edu).

Publisher Item Identifier S 0733-8716(98)05743-6.

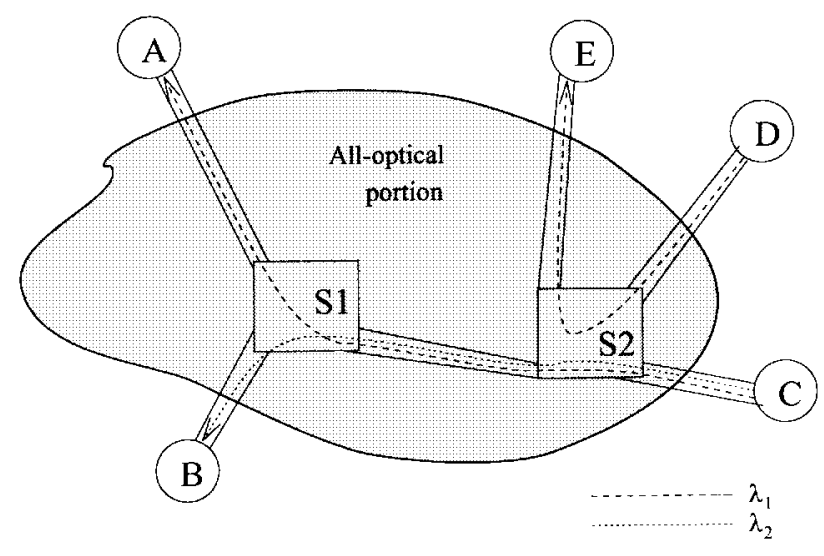

Fig. 1. An all-optical wavelength-routed network.

In order to transfer data from one access station to another, a connection needs to be set up at the optical layer similar to the case in a circuit-switched telephone network. This operation is performed by determining a path (route) in the network connecting the source station to the destination station and by allocating a common free (or idle) wavelength on all of the fiber links in the path. Such an all-optical path is referred to as a lightpath or a clear channel. The entire bandwidth available on this lightpath is allocated to the connection during its holding time during which the corresponding wavelength cannot be allocated to any other connection. When a connection is terminated, the associated lightpath is torn down, and the wavelength becomes idle once again on all of the links along the route.

Consider the network in Fig. 1. It shows a wavelengthrouted network containing two WDM crossconnects ( $\mathrm{S} 1$ and S2) and five access stations (A through E). Three lightpaths have been set up ( $\mathrm{C}$ to $\mathrm{A}$ on wavelength $\lambda_{1}, \mathrm{C}$ to $\mathrm{B}$ on $\lambda_{2}$, and $\mathrm{D}$ to $\mathrm{E}$ on $\lambda_{1}$ ). To establish any lightpath, we normally require that the same wavelength be allocated on all of the links in the path. This requirement is known as the wavelengthcontinuity constraint and wavelength-routed networks with this constraint are referred to as wavelength-continuous networks. The wavelength-continuity constraint distinguishes the wavelength-continuous network from a circuit-switched network which blocks calls only when there is no capacity along any of the links in the path assigned to the call. Consider the portion of the network in Fig. 2(a). Two lightpaths have been established in the network: 1) between node 1 and node 2 on wavelength $\lambda_{1}$ and 2) between node 2 and node 3 on wavelength $\lambda_{2}$. Now, suppose a lightpath between node 1 and node 3 must be set up. If there are only two wavelengths available in the network, establishing such a lightpath from node 1 to node 3 is now impossible even though there is 


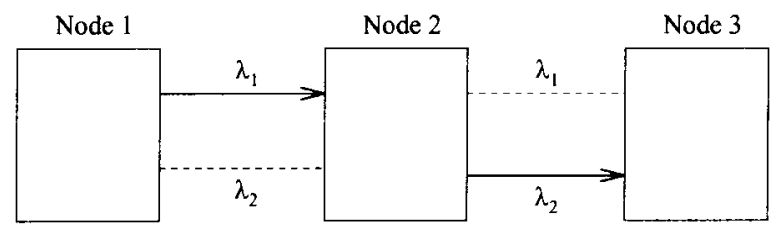

(a)

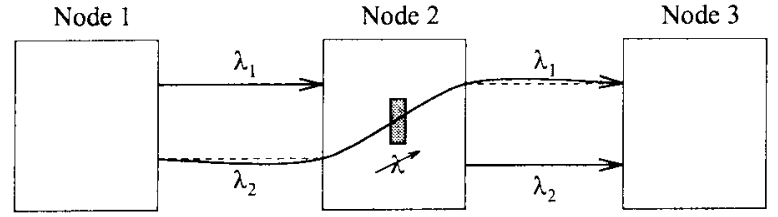

(b)

Fig. 2. Wavelength-continuity constraint in a wavelength-routed network: (a) without converter and (b) with converter.

a free wavelength on each of the links along the path from node 1 to node 3 . This is because the available wavelengths on the two links are different. Thus, a wavelength-continuous network may suffer from higher blocking as compared to a circuit-switched network.

It is easy to eliminate the wavelength-continuity constraint if we are able to convert the data arriving on one wavelength along a link into another wavelength at an intermediate node and forward it along the next link. Such a technique is feasible and is referred to as wavelength conversion and wavelength-routed networks with this capability are referred to as wavelength-convertible networks. A wavelength-convertible network which supports complete conversion at all nodes is functionally equivalent to a circuit-switched network, i.e., lightpath requests are blocked only when there is no available capacity on the path. In Fig. 2(b), a wavelength converter at node 2 is employed to convert data from wavelength $\lambda_{2}$ to $\lambda_{1}$. The new lightpath between node 1 and node 3 can now be established by using wavelength $\lambda_{2}$ on the link from node 1 to node 2 and then by using wavelength $\lambda_{1}$ to reach node 3 from node 2 . Notice that a single lightpath in such a wavelengthconvertible network can use a different wavelength along each of the links in its path. Thus, wavelength conversion may improve the efficiency in the network by resolving the wavelength conflicts of the lightpaths.

This study examines the role of wavelength converters in a wavelength-routed network. It includes a survey of the enabling device technologies as well as network design and analysis methodologies. This study also attempts to identify important unresolved issues in this field and to uncover challenging research problems. A note on terminology: wavelength converters have been referred to in the literature as wavelength shifters, wavelength translators, wavelength changers and frequency converters. This study will refer to these devices as wavelength converters.

This study is organized as follows (see Fig. 3). In Section II, the technologies which have made wavelength conversion possible are described. How wavelength converters are built and how switch designs have evolved to incorporate these converters will be the focus of this section. In Section III, the network design, control, and management issues involved in effectively using the technique of wavelength conversion are highlighted. The approaches taken to tackle some of these issues are described and new problems in this area are introduced. In Section IV, the benefits of wavelength conversion are described. Various approaches to quantify these benefits are discussed. Section V concludes this study.

\section{ENABLING TECHNOLOGIES}

Significant advances in optical and electronic device technologies have made wavelength conversion feasible [4]. Several different techniques have been demonstrated to perform wavelength conversion. A classification and comparison of these techniques is provided in Section II-A. Many novel switch designs have been proposed for utilizing these wavelength converters in a wavelength-convertible network. Some of these techniques are discussed in Section II-B.

\section{A. Wavelength-Converter Design}

The function of a wavelength converter is to convert data on an input wavelength onto a possibly different output wavelength among the $N$ wavelengths in the system (see Fig. 4). In this figure, and throughout this section, $\lambda_{s}$ denotes the input signal wavelength; $\lambda_{c}$, the output (converted) wavelength; $\lambda_{p}$, the pump wavelength; $f_{s}$, the input frequency; $f_{c}$, the converted frequency; $f_{p}$, the pump frequency; and $C W$, the continuous wave generated as the signal.

An ideal wavelength converter should possess the following characteristics [5]:

- transparency to bit rates and signal formats;

- fast setup time of output wavelength [6];

- conversion to both shorter and longer wavelengths;

- moderate input power levels;

- possibility for same input and output wavelengths (no conversion);

- insensitivity to input signal polarization;

- low-chirp output signal with high extinction ratio ${ }^{1}$ and large signal-to-noise ratio;

- simple implementation.

Our classification of the wavelength conversion techniques in this section follows that in [7]. Wavelength conversion techniques can be broadly classified into two types.

1) O/E Wavelength Conversion: In this method [8], the optical signal to be converted is first translated into the electronic domain using a photodetector (labeled $\mathrm{R}$ in Fig. 5). The electronic bit stream is stored in the buffer (labeled FIFO for the first-in-first-out queue mechanism). The electronic signal is then used to drive the input of a tunable laser (labeled T) tuned to the desired wavelength of the output (see Fig. 5, from [9]). This method has been demonstrated for bit rates up to $10-\mathrm{Gb} / \mathrm{s}$ [10]. This method, however, is much more complex and consumes much more power than the other methods described below [5]. Moreover, the process of O/E conversion adversely affects transparency. All information in the form of phase, frequency, and analog amplitude of the optical signal

\footnotetext{
${ }^{1}$ The extinction ratio is defined as the ratio of the optical power transmitted for a bit 0 to the power transmitted for a bit 1 .
} 


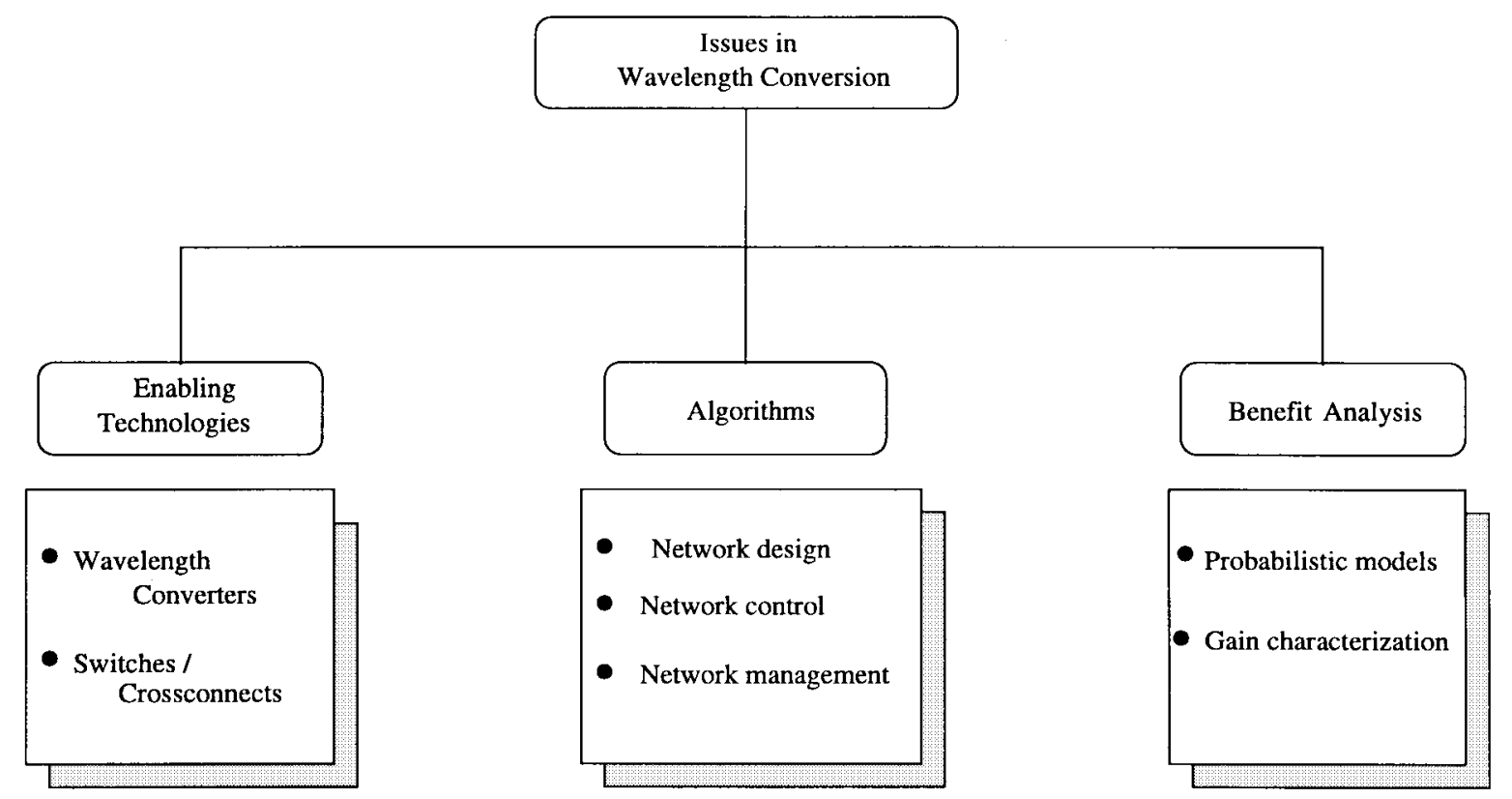

Fig. 3. Organization of this study.

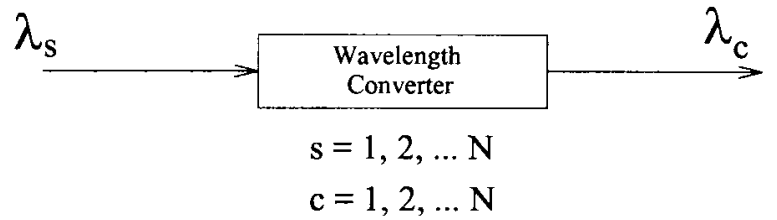

Fig. 4. Functionality of a wavelength converter.

is lost during the conversion process. The highest degree of transparency achievable is digital transparency, where digital signals of any bit rates up to a certain limit are accommodated [10].

2) All-Optical Wavelength Conversion: In this method, the optical signal is allowed to remain in the optical domain throughout the conversion process. Note that, in this context, all-optical, refers to the fact that there is no O/E conversion involved. Such all-optical methods can be further divided into the following categories and subcategories.

a) Wavelength conversion using wave-mixing: Wavemixing arises from a nonlinear optical response of a medium when more than one wave ${ }^{2}$ is present (see Fig. 6). It results in the generation of another wave whose intensity is proportional to the product of the interacting wave intensities. Wave-mixing preserves both phase and amplitude information, offering strict transparency. It also allows simultaneous conversion of a set of multiple input wavelengths to another set of multiple output wavelengths and could potentially accommodate signals with bit rates exceeding $100 \mathrm{~Gb} / \mathrm{s}$ [10]. In Fig. 6, the value $n=3$ corresponds to four-wave mixing (FWM) and $n=2$ corresponds to difference frequency generation. These techniques are described below.

- FWM: FWM (also referred to as four-photon mixing) is a third-order nonlinearity in silica fibers, which causes three

\footnotetext{
${ }^{2} \mathrm{~A}$ nonlinear response where new waves are generated can also happen with only a single input wave (e.g., Raman scattering).
}

optical waves of frequencies $f_{i}, f_{j}$ and $f_{k}(k \neq i, j)$ to interact in a multichannel WDM system [11] to generate a fourth wave of frequency given by

$$
f_{i j k}=f_{i}+f_{j}-f_{k} \text {. }
$$

Four-wave mixing is also achievable in an active medium such as a semiconductor optical amplifier (SOA). This technique provides modulation-format independence [12] and high bit-rate capabilities [13]. The conversion efficiency from pump energy to signal energy of this technique, however, is not very high and it decreases swiftly with increasing conversion span (shift between pump and output signal wavelengths) [14].

- Difference frequency generation (DFG): DFG is a consequence of a second-order nonlinear interaction of a medium with two optical waves: a pump wave and a signal wave. This technique offers a full range of transparency without adding excess noise to the signal, and spectrum inversion capabilities, but it suffers from low efficiency [10]. The main difficulties in implementing this technique lie in the phase matching of interacting waves and in fabricating a low-loss waveguide for high conversion efficiency [10]. In [15], a parametric wavelength interchanging crossconnect (WIXC) architecture has been proposed which uses DFG-based converters.

b) Wavelength conversion using cross modulation: These techniques utilize active semiconductor optical devices such as semiconductor optical amplifier (SOA's) and lasers. These techniques belong to a class known as optical-gating wavelength conversion [10].

- SOA's in cross-gain modulation (XGM) and cross-phase modulation (XPM) Mode: The principle behind using an SOA in the XGM mode is shown in Fig. 7 (from [5]). The intensity-modulated input signal modulates the gain in the SOA due to gain saturation. A continuous wave (CW) sig- 


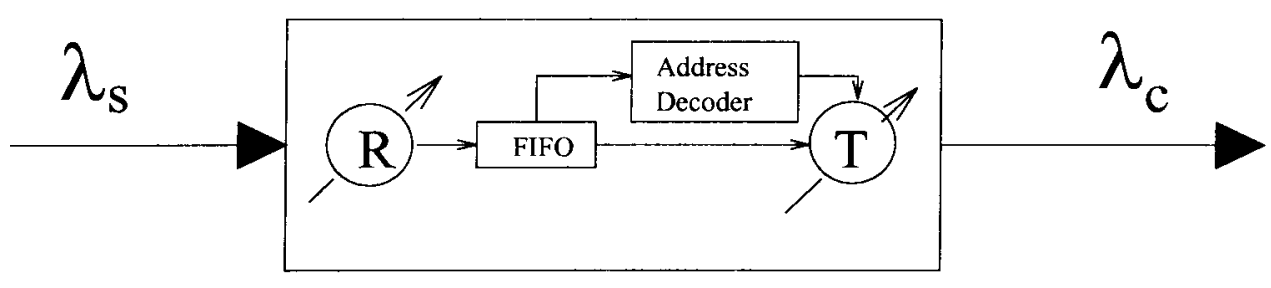

Fig. 5. An O/E wavelength converter.

nal at the desired output wavelength $\left(\lambda_{c}\right)$ is modulated by the gain variation so that it carries the same information as the original input signal. The input signal and the $\mathrm{CW}$ signal can be launched either co- or counterdirectionally into the SOA. The XGM scheme gives a wavelengthconverted signal that is inverted compared to the input signal. While the XGM scheme is simple to realize and offers penalty-free conversion at $10-\mathrm{Gb} / \mathrm{s}$ [5], it suffers from inversion of the converted bit stream and extinction ratio degradation for an input signal up-converted to a signal of equal or longer wavelength.

The operation of a wavelength converter using SOA in cross-phase modulation (XPM) mode is based on the fact that the refractive index of the SOA is dependent on the carrier density in its active region. An incoming signal that depletes the carrier density will modulate the refractive index and thereby result in phase modulation of a $\mathrm{CW}$ signal (wavelength $\lambda_{c}$ ) coupled into the converter [5], [16]. The SOA can be integrated into an interferometer so that an intensity-modulated signal format results at the output of the converter. Techniques involving SOA's in XPM mode have been proposed using the nonlinear optical loop mirror (NOLM) [17], Mach-Zender interferometer (MZI) [18], and Michelson interferometer (MI) [19]. Fig. 8 shows an asymmetric MZI wavelength converter based on SOA in XPM mode (from [5]). With the XPM scheme, the converted output signal can be either inverted or noninverted, unlike in the XGM scheme where the output is always inverted. The XPM scheme is also very power efficient compared to the XGM scheme [5]. Placing interferometric wavelength converters (IWC's) in the crossconnects has also been found to improve the transmission performance of an optical network by reducing the noise in the converted signal [20].

- Semiconductor lasers: Using single-mode semiconductor lasers, the lasing-mode intensity of the medium is modulated by input signal light through gain saturation. The output signal obtained is inverted compared to the input signal. This gain suppression mechanism has been employed in a distributed Bragg reflector (DBR) laser to convert signals at $10-\mathrm{Gb} / \mathrm{s}$ [21].

This section reviewed the various techniques used in the design of a wavelength converter. The actual choice of the technology to be employed in wavelength conversion in a network depends on the requirements of the particular system. It is clear, however, that opto-electronic converters offer only limited digital transparency. Moreover, deploying multiple opto-electronic converters in a WDM cross connect requires

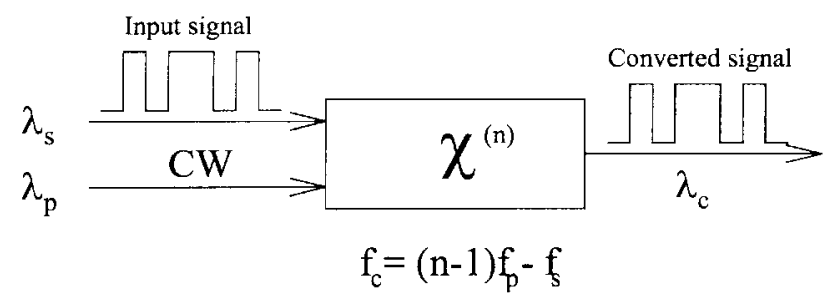

Fig. 6. A wavelength converter based on nonlinear wave-mixing effects.

sophisticated packaging to avoid cross talk among channels. This leads to increased costs-per-converter, further making this technology less attractive than all-optical converters [10]. Other disadvantages of opto-electronic converters include complexity and large power consumption [5]. All-optical converters based on SOA's using the XGM and the XPM conversion schemes at present seem well suited for system use. Converters based on four-wave mixing, though transparent to different modulation formats, perform inefficiently [5]. Wave-mixing converters, however, are the only category of wavelength converters that offer the full range of transparency, while also allowing the simultaneous conversion of a set of input wavelengths to another set of output wavelengths. In this respect, different frequency-generation-based methods offer great promise. Further details on comparison of various wavelength conversion techniques can be found in [5], [7], [10], [22], and [23].

The next section will examine various switch architectures which have been proposed in the literature for use in a wavelength-convertible network.

\section{B. Wavelength-Convertible Switch Design}

As wavelength converters become readily available, a vital question comes to mind: Where do we place them in the network? An obvious location is in the switches (or cross connects) in the network. A possible architecture of such a wavelength-convertible switching node is the dedicated wavelength convertible switch (see Fig. 9, from [24]). In this architecture, each wavelength along each output link in a switch has a dedicated wavelength converter, i.e., an $M \times M$ switch in an $N$-wavelength system requires $M \times N$ converters. The incoming optical signal from a fiber link at the switch is first wavelength-demultiplexed into separate wavelengths. Each wavelength is switched to the desired output port by the nonblocking optical switch. The output signal may have its wavelength changed by its wavelength converter. Finally, various wavelengths are multiplexed to form an aggregate signal coupled to an outbound fiber link. 


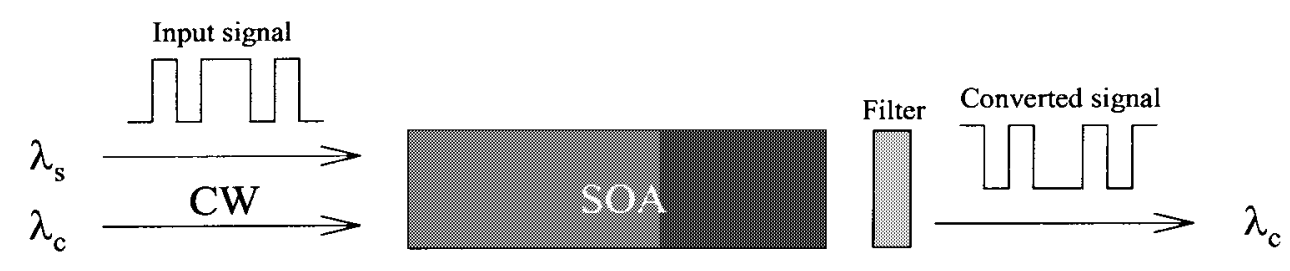

Fig. 7. A wavelength converter based on XGM in an SOA.

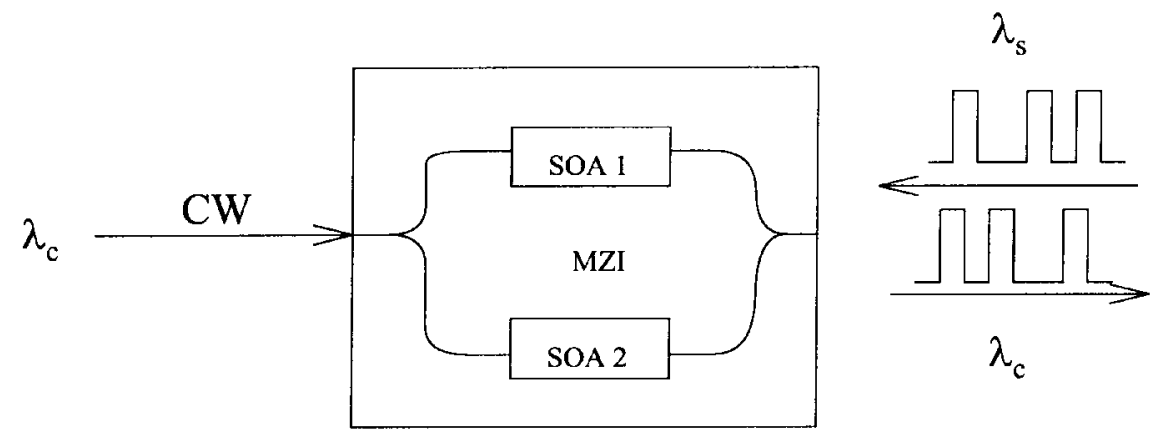

Fig. 8. An interferometric wavelength converter based on XPM in SOA's.

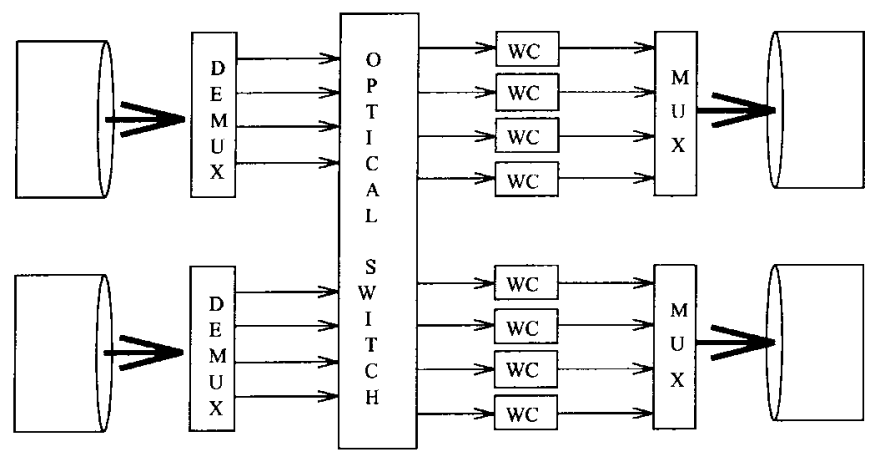

Fig. 9. A switch which has dedicated converters at each output port for each wavelength (WC denotes a wavelength converter).

The dedicated wavelength-convertible switch, however, is not very cost efficient since all of the wavelength converters may not be required all the time [25]. An effective method to cut costs is to share the converters. Two architectures have been proposed for switches sharing converters [24]. In the share-per-node structure [see Fig. 10(a)], all the converters at the switching node are collected in a converter bank. (A converter bank is a collection of a few wavelength converters each of which is assumed to have identical characteristics and can convert any input wavelength to any output wavelength.) This bank can be accessed by any of the incoming lightpaths by appropriately configuring the larger optical switch in Fig. 10(a). In this architecture, only the wavelengths which require conversion are directed to the converter bank. The converted wavelengths are then switched to the appropriate outbound link by the second (small) optical switch. In the share-per-link structure [see Fig. 10(b)], each outgoing link is provided with a dedicated converter bank which can be accessed only by those lightpaths traveling on that particular outbound fiber link. The optical switch can be configured appropriately to direct wavelengths toward a particular link, either with or without conversion.

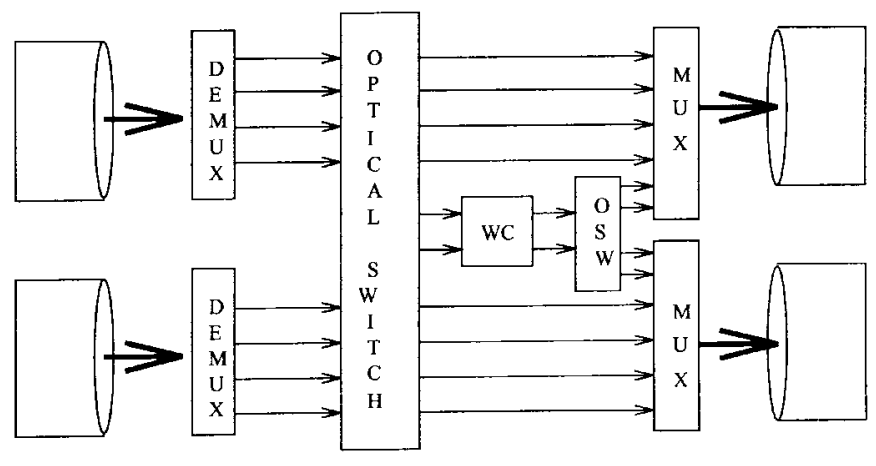

(a)

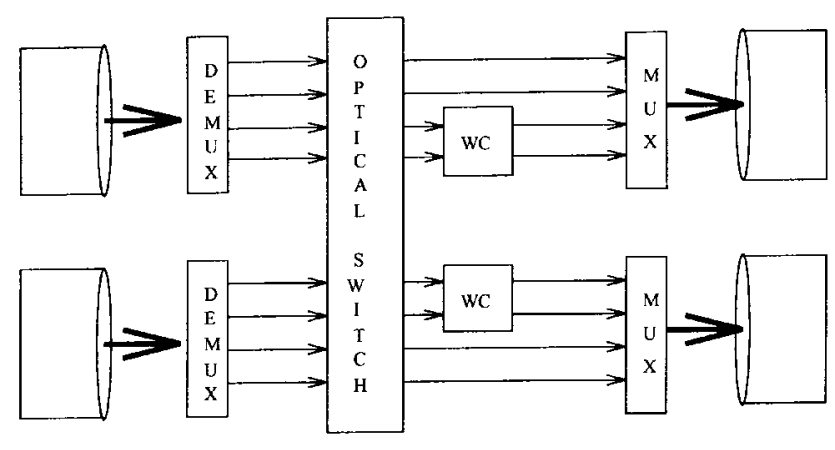

(b)

Fig. 10. Switches which allow sharing of converters. (a) Share-per-node wavelength-convertible switch architecture. (b) Share-per-link wavelength-convertible switch architecture.

When $\mathrm{O} / \mathrm{E}$ wavelength conversion is used, the functionality of the wavelength converter can be performed at the access stations instead of at the switches. The share-with-local switch architecture proposed in [24] (see Fig. 11) and the simplified network access station architecture proposed in [26] (see Fig. 12) fall under this category. 


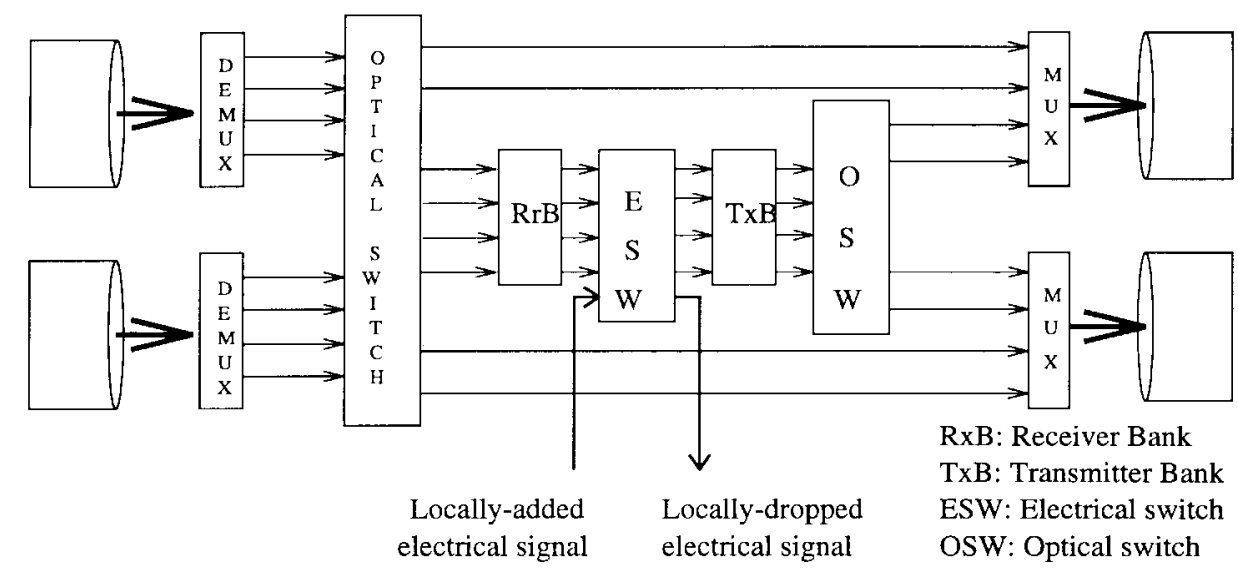

Fig. 11. The share-with-local wavelength-convertible switch architecture.

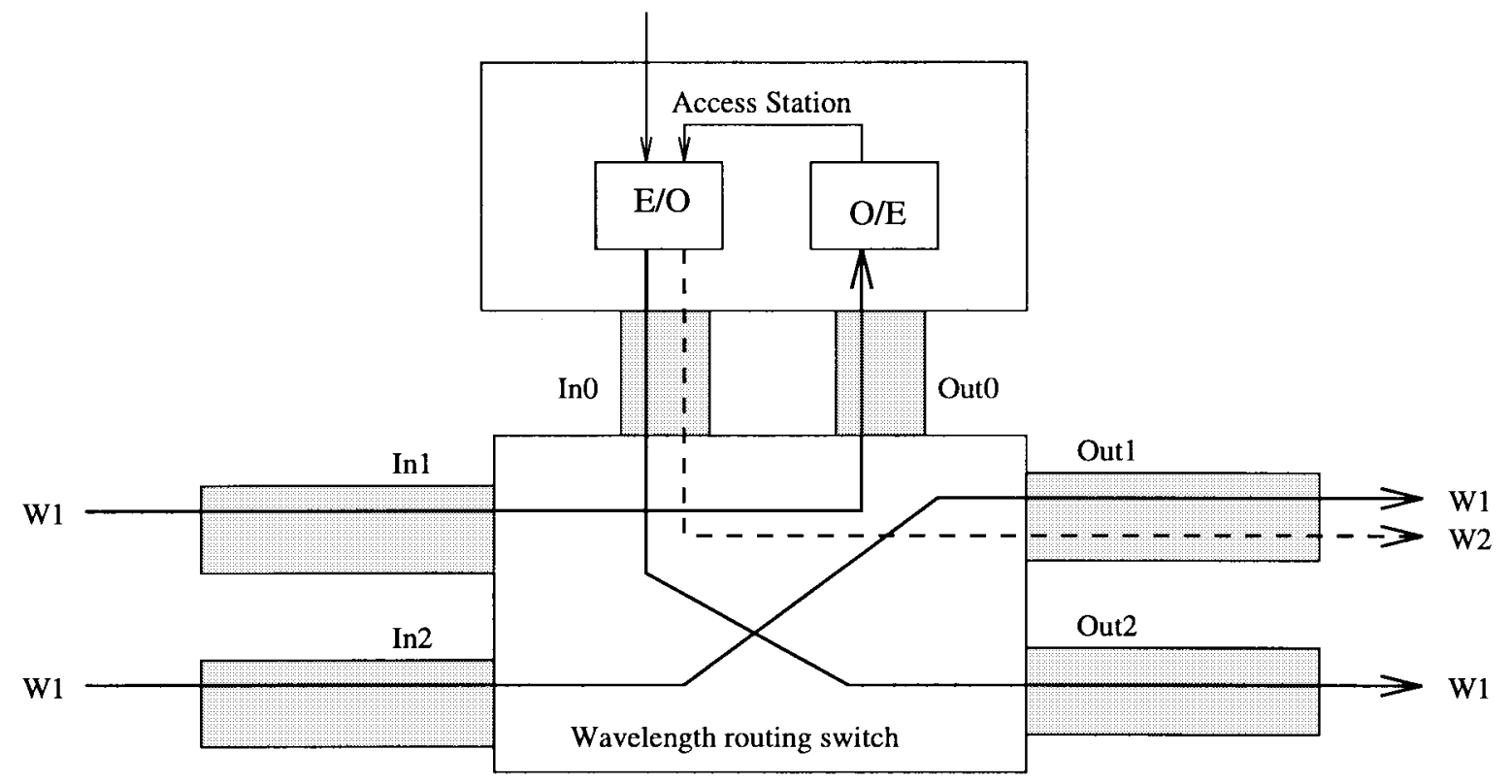

Fig. 12. Architecture which supports electronic wavelength conversion.

\section{Network Design, CONTROL, AND MANAGEMENT ISSUES}

\section{A. Network Design}

Network designs must evolve to effectively incorporate wavelength conversion. Network designers must choose not only among the various conversion techniques described in Section II-A, but also among the several switch architectures described in Section II-B. An important challenge in the design is to overcome the limitations in using wavelength-conversion technology. These limitations fall into the following three categories.

1) Sparse location of wavelength converters in the network: As long as wavelength converters remain expensive [10], it may not be economically viable to equip all the nodes in a WDM network with these devices. The effects of sparse conversion (i.e., having only a few converting switches in the network) on lightpath blocking have been examined in [27]. An interesting question which has not been answered thoroughly is where (optimally?) to place these few converters in an arbitrary network and what is the likely upgrade-path toward full fledged convertibility? A heuristic technique for the placement of these sparse converters in an all-optical network is presented in [25].

2) Sharing of converters: Even among the switches capable of wavelength conversion, it may not be cost-effective to equip all the output ports of a switch with this capability. Designs of switch architectures have been proposed (see Section II-B) which allow sharing of converters among the various signals at a switch. It has been shown in [24] that the performance of such a network saturates when the number of converters at a switch increases beyond a certain threshold. An interesting problem is to quantify the dependence of this threshold on the routing algorithm used and the blocking probability desired.

3) Limited-range wavelength conversion: Four-wavemixing-based all-optical wavelength converters provide only a limited-range conversion capability. If the range is limited to $k$, then an input wavelength $\lambda_{i}$ can 
only be converted to wavelengths $\lambda_{\max (i-k, 1)}$ through $\lambda_{\min (i+k, N)}$, where $N$ is the number of wavelengths in the system (indexed 1 through $N$ ). Analysis shows that networks employing such devices, however, compare favorably with those utilizing converters with full-range capability, under certain conditions [28]. Limited-range wavelength conversion can also be provided at nodes using O/E conversion techniques [29].

Other wavelength-converter techniques have some limitations too. As seen in Section II-A, the wavelength converter using SOA's in XGM mode suffers greater degradation when the input signal is up-converted to a signal of equal or longer wavelength than when it is down-converted to a shorter wavelength. Moreover, since the signal quality usually worsens after multiple such conversions, the effect of a cascade of these converters can be substantial. The implications of such a device on the design of the network need to studied further.

Apart from efficient wavelength-convertible switch architectures and their optimal placement, several other design techniques offer promise. Networks equipped with multiple fibers on each link have been considered for potential improvement [30] in wavelength-convertible networks and suggested as a possible alternative to conversion. This work will be reviewed in greater detail in Section IV. Another important problem is the design of a fault-tolerant wavelength-convertible network [31]. Such a network could reserve capacity on the links to handle disruptions due to link failure caused by a cut in the fiber. Quantitative comparisons need to be developed for the suitability of a wavelength-convertible network in such scenarios.

\section{B. Network Control}

Control algorithms are required in a network to manage its resources effectively. An important task of the control mechanism is to provide routes (i.e., sets of fiber links) to the lightpath requests and to assign wavelengths on each of the links along this route while maximizing a desired system parameter, e.g., throughput. Such routing and wavelength assignment (RWA) schemes can be classified into static and dynamic categories, depending on whether the lightpath requests are known a priori or not. These two categories are described below.

1) Dynamic routing and wavelength assignment: In a wavelength-routed optical network, lightpath requests between source-destination pairs arrive at random and each lightpath has a random holding time after which it is torn down. These lightpaths need to be set up dynamically by determining a route through the network connecting the source to the destination and assigning a free wavelength along this path. Two lightpaths which have at least one link in common cannot use the same wavelength. Moreover, the same wavelength has to be assigned to a path on all of its links. This is the wavelength-continuity constraint described in Section I. This routing and wavelength assignment (RWA) problem, or variants of it, has been studied earlier [32]-[34] for networks without wavelength conversion.
Dynamic routing algorithms have been proposed for use in a wavelength-convertible network [24], [35]. Algorithms have also been studied which use a fixed-path or deterministic routing [36]. In such a scheme, there is a fixed path between every sourcedestination pair in the network. Several RWA heuristics have been designed based on which wavelength to assign to a lightpath along the fixed path and which, if any, lightpaths to block selectively [32]. However, design of efficient routing algorithms which incorporate the limitations in Section III-A still remains an open problem.

2) Static RWA: In contrast to the dynamic routing problem described above, the static RWA problem assumes that all the lightpaths that are to be set up in the network are known initially. The objective is to maximize the total throughput in the network, i.e., the total number of lightpaths which can be established simultaneously in the network. An upper bound on the carried traffic per available wavelength has been obtained (for a network with and without wavelength conversion) by relaxing the corresponding integer linear program (ILP) [36]. Several heuristic-based approaches have been proposed for solving the static RWA problem in a network without wavelength conversion [33], [37]. A special case of the static RWA problem, in which all the lightpath requests can be accommodated, is discussed in [29] for networks with limited wavelength conversion. Again, efficient algorithms which incorporate the limitations in Section III-A for a wavelength-convertible network are still unavailable.

\section{Network Management}

Wavelength conversion may be used to promote interoperability across subnetworks which are managed independently. Thus, it supports the distribution of network control and management functionalities among smaller subnetworks by allowing flexible wavelength assignments within each subnetwork [10], [38]. As shown in Fig. 13, network operators 1, 2, and 3 manage their own subnetworks and may use wavelength conversion for communication across subnetworks. In [39], the authors propose to install wavelength converters at the borders between nonoverlapping network partitions in the Cost 239 European optical network.

\section{BENEFIT ANALYSIS}

As mentioned above, the availability of full wavelength conversion simplifies the management of the network-the wavelength-assignment algorithm in such a network becomes simpler because all the wavelengths can be treated equivalently, and wavelengths used on successive links along a path can be independent of one another. The benefits, however, of wavelength conversion in reducing blocking and improving other performance metrics are not nearly as universal or apparent. While full wavelength conversion eliminates the wavelength-continuity constraint (see Section I), the actual performance benefits available in a typical network are found 


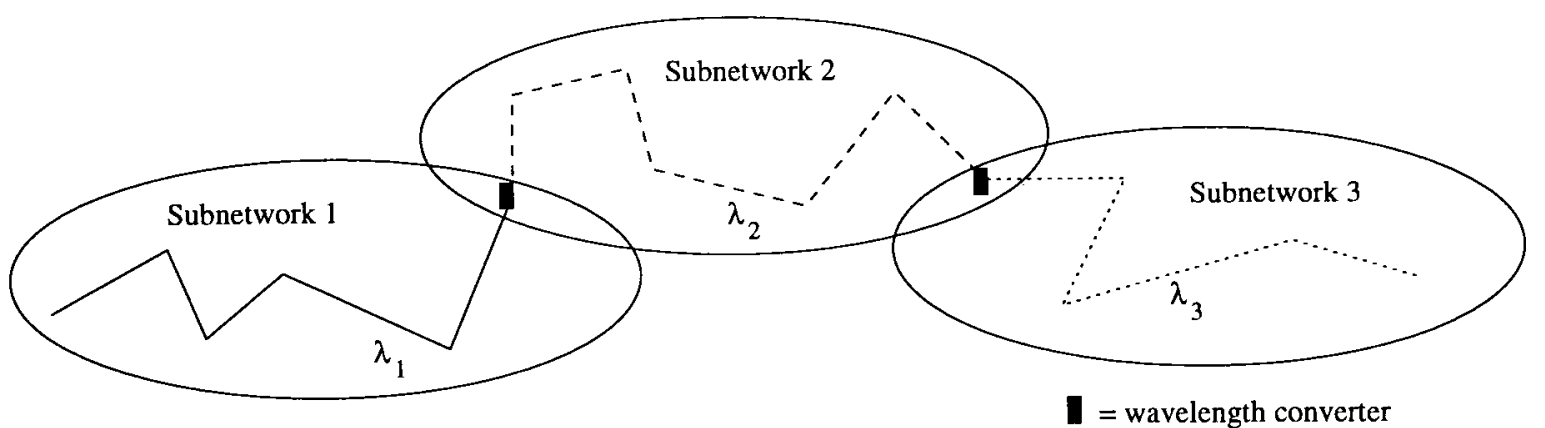

Fig. 13. Wavelength conversion for distributed network management.

TABLE I

COMPARISON OF ANALYTICAL Models

\begin{tabular}{l|c|c|c|c}
\hline & $\begin{array}{c}\text { Kovačević et al. } \\
{[40]}\end{array}$ & $\begin{array}{c}\text { Subramaniam } \text { et al. } \\
{[27]}\end{array}$ & $\begin{array}{c}\text { Birman } \\
{[43]}\end{array}$ & $\begin{array}{c}\text { Barry et al. } \\
{[44]}\end{array}$ \\
\hline Traffic & Dynamic & Dynamic & Dynamic & Steady-state \\
\hline Arrival Process & Poisson & Poisson & Poisson & Unspecified \\
\hline Holding Time & Exponential & Exponentia] & Exponential & Unspecified \\
\hline Routing & Fixed & Fixed & Fixed, & Fixed \\
Weast-loaded & Random & Random \\
$\begin{array}{l}\text { Assignment } \\
\text { Link Loads }\end{array}$ & Independent & $\begin{array}{c}\text { Correlated } \\
\text { (Markovian) }\end{array}$ & Dependent & $\begin{array}{c}\text { Correlated } \\
\text { (Markovian) }\end{array}$ \\
\hline $\begin{array}{l}\text { Wavelengths on } \\
\text { adjacent links }\end{array}$ & Independent & Dependent & Independent & Dependent \\
\hline $\begin{array}{l}\text { Performance } \\
\text { Metric }\end{array}$ & Blocking & Blocking & Blocking & Wavelength \\
\hline $\begin{array}{l}\text { Computational } \\
\text { Complexity }\end{array}$ & Mobability $\left(p_{b}\right)$ & probability $\left(p_{b}\right)$ & probability $\left(p_{b}\right)$ & utilization gain $(G)$ \\
\hline
\end{tabular}

to depend on factors such as connectivity and traffic load. Using analytical models and simulations, efforts have been made to quantify these benefits in typical networks. We present a review of several of these studies in the remainder of this section.

\section{A. Analytical Models}

Analytical models have been developed by several researchers to quantify the benefits of wavelength converters. We present below a brief review of the fundamental models available today. All of the models discussed below make the following assumptions.

- Each lightpath uses the entire bandwidth of a wavelength channel.

- Each fiber link has the same number $(W)$ of wavelengths.

- Each station has arrays of $W$ transmitters and $W$ receivers.

- The network only supports point-to-point traffic, i.e., there is no multicasting.

- Connection requests are not queued, i.e., if a connection is blocked, it is immediately discarded.
The main differences between the models are highlighted in Table I. Below, we describe these approaches in detail.

1) A Probabilistic Model with Independent Link-Load Assumption [40]:

Overview: An approximate analytical model is developed for a fixed-path (deterministic) routed network with an arbitrary topology, both with and without wavelength conversion. This model is then used along with simulations to study the performance of three example networks: the nonblocking centralized switch, the two-dimensional torus network, and the ring network. The traffic loads and the wavelength-occupancy probabilities on the links are both assumed to be independent. A wavelength-assignment strategy is employed in which a lightpath is assigned a wavelength at random from among the available wavelengths in the path. The blocking probability of the lightpaths is used to study the performance of the network. The benefits of wavelength conversion are found to be modest in the nonblocking centralized switch and the ring; however, wavelength conversion is found to significantly improve the performance of a large two-dimensional torus network. The analytical model employed in this study cannot be applied to a ring network because the very high load correlation along the 
links of a path in a ring network invalidates the independent link-load assumption.

Details: First, we consider the case when there is no wavelength conversion in the network. In this case, a connection request is blocked when there is no wavelength available on every link of the path. The approach in [40] determines the conditional probability that $k$ wavelengths are available for a connection on a two-hop path and extends the analysis for an $n$-hop path.

Let $W$ be the number of wavelengths per fiber $T$ the average duration of a connection and $\lambda_{i}$ the arrival rate on the $i$ th link of the path. $L_{i}$, the average offered load on the $i$ th link of the path, is then given by $L_{i}=\lambda_{i} T$. Let $p_{k}^{(i)}$ be the probability that $k$ wavelengths are used on the $i$ th link of the path. Assuming Poisson arrivals on the link and exponential holding times, we have

$$
p_{k}^{(i)}=\frac{\left(\lambda_{i} T\right)^{k}}{k !} p_{0}^{(i)}=\frac{\frac{L_{i}^{k}}{k !}}{\sum_{l=0}^{W} \frac{L_{i}^{l}}{l !}} .
$$

For a connection requiring a single hop, the blocking probability is equal to $p_{W}^{(1)}$, the probability that all $W$ wavelengths are busy on the link connecting the source and the destination. Let $q_{k}^{(n)}$ be the probability that there are $k$ busy wavelengths over the first $n$ hops of the path. For a one-hop connection, we have $q_{k}^{(1)}=p_{k}^{(1)}, k \in 1, \cdots, W$. For a two-hop path, the conditional probability that there are $k$ wavelengths available for a connection, given that $n_{a}$ and $n_{b}$ wavelengths are free on links $a$ and $b$ (assuming that the distributions of assigned wavelengths at links $a$ and $b$ are mutually independent) equals

$$
R\left(k \mid n_{a}, n_{b}\right)=\frac{\left(\begin{array}{c}
n_{a} \\
k
\end{array}\right)\left(\begin{array}{c}
W-n_{a} \\
n_{b}-k
\end{array}\right)}{\left(\begin{array}{c}
W \\
n_{b}
\end{array}\right)}
$$

if $\max \left(0, n_{a}+n_{b}-W\right) \leq k \leq \min \left(n_{a}, n_{b}\right)$ and is equal to zero otherwise. Using this conditional probability, the distribution of busy wavelengths over the two-hop path follows:

$$
q_{k}^{(2)}=\sum_{i=0}^{W} \sum_{j=0}^{W} R(W-k \mid W-i, W-j) p_{i}^{(1)} p_{j}^{(2)} .
$$

The blocking probability for a two-hop connection is thus $P^{(2)}=q_{W}^{(2)}$. Hence, for a $n$-hop path, we have (using recursion)

$$
q_{k}^{(n)}=\sum_{i=0}^{W} \sum_{j=0}^{W} R(W-k \mid W-i, W-j) q_{i}^{(n-1)} p_{j}^{(n)}
$$

and

$$
P^{(n)}=q_{W}^{(n)} .
$$

Next, we consider the case when wavelength conversion is available in the network. Note that a lightpath is blocked only when one or more links on the path have all of their wavelengths occupied. Thus, the blocking probability for an $n$-hop connection equals

$$
P^{(n)}=1-\prod_{i=1}^{n}\left(1-p_{W}^{(i)}\right) .
$$

The above analysis (for the path-blocking probabilities) assumes that the link-loads along the path are already known. In practice, however, it is the traffic matrix (which represents the offered load between a pair of stations), which is usually known and not the link-loads. Hence, the authors estimated the link-loads in the network using an approach similar to that in [41]. For a network with wavelength conversion, the arrival process on a link $s$ is independent of the number of the connections carried by the link (assuming independent linkloads). Thus, the arrivals on the link can be considered to be Poisson arrivals, and the number of occupied wavelengths can be represented by the distribution given in (1). To make the analysis of the network without wavelength conversion tractable, however, the approach in [40] makes an approximation by assuming Poisson arrivals at the links in this case also. The network-blocking probabilities can be obtained by solving the set of coupled nonlinear equations called Erlang's map. It is shown in [41] that this set of equations has a unique solution for the network with wavelength conversion. The authors provide an iterative procedure to solve these equations and compute the blocking probability for the network without wavelength conversion.

\section{2) Sparse Wavelength Conversion [27]:}

Overview: Sparse wavelength conversion, in which only a few nodes in the network are equipped with wavelength converters (see Section III-A) is studied in [27]. ${ }^{3}$ Two different switching nodes are available in the network-nodes with no wavelength conversion capability and nodes which can convert any incoming wavelength to an arbitrary outgoing wavelength. An analytical model for evaluating the pathblocking performance of such networks is also presented and is shown to be accurate for a variety of network topologies. The model improves on the one in [40] by relaxing the independence assumptions on the loads and wavelength-occupancy probabilities of the links. The authors find that the usefulness of wavelength converters depends on the connectivity of the network. Converters are not very useful in networks with low connectivity, such as the ring, because of the high load correlation between links. Moreover, converters are also found to be of little use in networks with high connectivity, such as the hypercube, because of the small hop-lengths. Converters, however, offer significant benefits in networks with medium connectivity, such as the mesh-torus network, because the linkload correlations are low while the hop-lengths are large. The authors show that, in most cases, only a small fraction of the nodes has to be equipped with wavelength conversion capability for good performance.

Details: In their model, the authors incorporate the load correlation among the links in the network. In particular, they assume that the load on link $i$ of a path given the loads on links

\footnotetext{
${ }^{3}$ Networks without wavelength conversion and those with full wavelength conversion are handled as extreme cases under the same framework of sparse conversion.
} 
$1,2, \cdots, i-1$, depends only on the load on link $i-1$. Hence, their analytical model is a Markovian correlation model. First, they derive the conditional free-wavelength distribution on a two-hop path using the following notations:

- $Q\left(w_{f}\right)=\operatorname{Pr}\left\{w_{f}\right.$ wavelengths are free on a link $\}$;

- $S\left(y_{f} \mid x_{p f}\right)=\operatorname{Pr}\left\{y_{f}\right.$ wavelengths are free on a link of a path $\mid x_{p f}$ wavelengths are free on the previous link of the path\};

- $U\left(z_{c} \mid y_{f}, x_{p f}\right)=\operatorname{Pr}\left\{z_{c}\right.$ lightpaths continue to the current link from the previous link of a path $\mid x_{p f}$ wavelengths are free on the previous link, and $y_{f}$ wavelengths are free on the current link\};

- $R\left(n_{f} \mid x_{f f}, y_{f}, z_{c}\right)=\operatorname{Pr}\left\{n_{f}\right.$ wavelengths are free on a two-hop path $\mid x_{f f}$ wavelengths are free on the first hop of the path, $y_{f}$ wavelengths are free on the second hop, and $z_{c}$ lightpaths continue from the first to the second hop $\}$;

- $T_{n f}^{(l)}, y_{f}=\operatorname{Pr}\left\{n_{f}\right.$ wavelengths are free on an $l$-hop path and $y_{f}$ wavelengths are free on hop $\left.l\right\}$;

- $p_{l}=\operatorname{Pr}\{$ an $l$-hop path is chosen for routing $\}$.

Now consider a two-hop path between nodes 0 and 2 passing through node 1 . Let $C_{l}$ be the number of lightpaths that enter the path at node 0 and leave at node 1 , let $C_{c}$ be the number of lightpaths that enter the path at node 0 and continue on to the second link, and let $C_{n}$ be the number of lightpaths that enter the path at node 1 and let $\lambda_{l}, \lambda_{c}$, and $\lambda_{n}$ be the corresponding lightpath arrival rates. Then, the number of lightpaths that use the first link is $C_{l}+C_{c}$ and the number of lightpaths that use the second link is $C_{c}+C_{n}$. By the assumption of uniform traffic distribution, the arrival rate of lightpaths that enter the path at node 1 is the same as the arrival rate of lightpaths that leave the path at node 1 , i.e., $\lambda_{l}=\lambda_{n}$. The quantities $C_{l}, C_{c}$, and $C_{n}$ can therefore be characterized by a three-dimensional Markov chain, with each state represented by an integer triplet $\left(c_{l}\right.$, $\left.c_{c}, c_{n}\right)$. The probabilities $R\left(n_{f} \mid x_{f f}, y_{f}, z_{c}\right), U\left(z_{c} \mid y_{f}, x_{p f}\right)$, $S\left(y_{f} \mid x_{p f}\right)$, and $Q\left(w_{f}\right)$ are then derived for the two-hop path. The authors then extend the analysis to determine the blocking probability on a path of arbitrary hop length.

In order to keep the analysis simple, the authors assume that the effect of lightpath blocking on the carried load along the links is negligible. This assumption, which is valid only for low blocking probabilities, means that the entire load offered to the network is carried along the links. From the lightpath arrival rates at nodes, an approximation for the link arrival rates $\lambda_{l}$ and $\lambda_{c}$ can be found as follows. Let $N$ be the number of nodes in the network, $\lambda$ be the lightpath arrival rate at a node, and $\bar{H}$ be the average hop distance. Then the average lightpath arrival rate per link $(\gamma)$ is given by

$$
\gamma=\frac{N \lambda \bar{H}}{L}
$$

Suppose there are $k$ exit links per node and that, if a lightpath does not leave the network at a node, it chooses one of the $k$ exit links arbitrarily. Then, the arrival rate of lightpaths that continue on to the next link of a path can be estimated as

$$
\lambda_{c}=\gamma \frac{1-1 / \bar{H}}{k}
$$

from which we have

$$
\lambda_{l}=\gamma-\lambda_{c}
$$

The parameter $q$, the conversion density of the network, is used to model a network with sparse wavelength conversion. The number of converter nodes in an $N$-node network is binomially distributed with an average of $N q$ converters. The blocking probability in a network with sparse wavelength conversion is then computed recursively by conditioning on the event that node $i$ is the last converter on a $l$-hop path in the network $(1 \leq i \leq(l-1))$.

3) A Probabilistic Model for a Class of Networks [43]:

Overview: This study provides an approximate method for calculating the blocking probability in a wavelengthrouted network. The model considers Poisson input traffic and uses a Markov chain model with state-dependent arrival rates. Two different routing schemes are considered: fixed routing, where the path from a source to a destination is unique and is known beforehand, and least loaded routing (LLR), an alternate-path scheme where the route from source to destination is taken along the path which has the largest number of idle wavelengths. Analysis and simulations are carried out using fixed routing for networks of arbitrary topology with paths of at most three-hops-length, and using LLR for fully connected networks with paths of one or two hops. The blocking probability is found to be larger without wavelength conversion. This method is, however, computationally intensive and is tractable only for densely connected networks with a few nodes.

Details: We consider a network of arbitrary topology with $J$ links and $C$ wavelengths on each link. A route $R$ is a subset of links from $\{1,2, \cdots, J\}$. Lightpath requests arrive for route $R$ as a Poisson stream with rate $\alpha_{R}$. A lightpath for route $R$ is set up if there is a wavelength $w_{i}$ such that $w_{i}$ is idle on all links of route $R$. The holding times of all lightpaths are exponentially distributed with unit mean.

Let $X_{R}$ be the random variable denoting the number of idle wavelengths on route $R$. If $R=\{i, j, k\}$, then we may write $X_{R}$ as $X_{i, j, k}$. Let $\mathbf{X}=\left(X_{1}, X_{2}, \cdots, X_{J}\right)$ and let

$$
q_{j}(m)=\operatorname{Pr}\left[X_{j}=m\right], \quad m=0,1, \cdots, C
$$

be the idle capacity distribution on link $j$. The author assumes that the random variables $X_{j}$ are independent as in [42]. Then

$$
q(m)=\prod_{j=1}^{J} q_{j}\left(m_{j}\right)
$$

where $\mathbf{m}=\left(m_{1}, m_{2}, \cdots, m_{J}\right)$. Further, the author assumes that, given $m$ idle wavelengths on link $j$, the time until the next lightpath is set up on link $j$ is exponentially distributed with parameter $\alpha_{j}(m)$. It follows that the number of idle wavelengths on link $j$ can be viewed as a birth-death process so that

$$
q_{j}(m)=\frac{C(C-1) \cdots(C-m+1)}{\alpha_{j}(1) \alpha_{j}(2) \cdots \alpha_{j}(m)} q_{j}(0)
$$


where

$$
q_{j}(0)=\left[1+\sum_{m=1}^{C} \frac{C(C-1) \cdots(C-m+1)}{\alpha_{j}(1) \alpha_{j}(2) \cdots \alpha_{j}(m)}\right]^{-1} .
$$

$\alpha_{j}(m)$ is obtained by combining the contributions from the request streams to routes which have link $j$ as a member, as follows:

$$
\begin{aligned}
\alpha_{j}(m) & =0, \quad \text { if } m=0, \\
& =\sum_{R: j \in R} a_{R} \operatorname{Pr}\left[X_{R}>0 \mid X_{j}=m\right], \\
& \text { if } m=1,2, \cdots, C .
\end{aligned}
$$

The blocking probability for lightpaths to route $R\left(L_{R}=\right.$ $\left.\operatorname{Pr}\left[X_{R}=0\right]\right)$ is then calculated for routes up to three hops. Similarly, for the case of LLR, the author derives the blocking probability $\left(L_{R}\right)$ in a fully connected network.

4) A Probabilistic Model Without Independent Link-Load Assumption [44]:

Overview: A model which is more analytically tractable than the ones in [43] and [40] is provided in [44]; however, it uses more simplistic traffic assumptions. The link-loads are not assumed to be independent; however, the assumption is retained that a wavelength is used on successive links independent of other wavelengths. The concept of interference length $(L)$, i.e., the expected number of links shared by two lightpaths which share at least one link, is introduced. Analytical expressions for the link utilization and the blocking probability are obtained by considering an average path which spans average hop distance $(H)$ links in networks with and without wavelength conversion. The gain $(G)$ due to wavelength conversion is defined as the ratio of the link utilization with wavelength conversion to that without wavelength conversion for the same blocking probability. The gain is found to be directly proportional to the effective path length $(H / L)$. A larger switch size $(\Delta)$ tends to increase the blocking probability in networks without wavelength conversion. The model used in [44] is also applicable to ring networks, unlike the work in [40], and it correctly predicts the low gain in utilizing wavelength conversion in ring networks.

Details: The simplified model described initially in [44] is based on standard series independent link assumptions, i.e., a lightpath request sees a network in which a wavelength's usage on a fiber link is statistically independent of other fiber links and other wavelengths.

This model, however, generally tends to over-estimate the blocking probability. ${ }^{4}$

Let there be $W$ wavelengths per fiber link, and let $\rho$ be the probability that a wavelength is used on any fiber link. (Since $\rho W$ is the expected number of busy wavelengths on any fiber link, $\rho$ is also the fiber utilization of any fiber.) We will consider a $H$-link path for a connection from node $A$ to node $B$ that needs to be set up.

First, let us consider a network with wavelength converters. The probability $P_{b}^{\prime}$ that the connection request from $A$ to $B$

\footnotetext{
${ }^{4}$ The link-load independence assumption is relaxed later on in [44] to provide a more accurate model.
}

will be blocked equals the probability that, along this $H$-link path, there exists a fiber link with all of its $W$ wavelengths in use, so that

$$
P_{b}^{\prime}=1-\left(1-\rho^{W}\right)^{H} .
$$

Defining $q$ to be the achievable utilization for a given blocking probability in a wavelength-convertible network, we have

$$
q=\left[1-\left(1-P_{b}^{\prime}\right)^{1 / H}\right]^{1 / W} \approx\left(\frac{P_{b}^{\prime}}{H}\right)^{1 / W}
$$

where the approximation holds for small values of $P_{b}^{\prime} / H$.

Next, let us consider a network without wavelength converters. The probability $P_{b}$ that the connection request from $A$ to $B$ will be blocked equals the probability that, along this $H$-link path, each wavelength is used on at least one of the $H$ links, so that

$$
P_{b}=\left[1-(1-\rho)^{H}\right]^{W} .
$$

Defining $p$ to be the achievable utilization for a given blocking probability in a network without wavelength conversion, we have

$$
p=1-\left(1-P_{b}^{1 / W}\right)^{1 / H} \approx-\frac{1}{H} \ln \left(1-P_{b}^{1 / W}\right)
$$

where the approximation holds for large values of $H$, and for $P_{b}^{1 / W}$ not too close to unity. Observe that the achievable utilization is inversely proportional to $H$, as expected.

We define $G=q / p$ to be a measure of the benefit of wavelength conversion, which is the increase in (fiber or wavelength) utilization for the same blocking probability. From (16) and (18), after setting $P_{b}=P_{b}^{\prime}$, we get

$$
G \approx H^{1-(1 / W)} \frac{P_{b}^{1 / W}}{-\ln \left(1-P_{b}^{1 / W}\right)}
$$

where the approximation holds for small $P_{b}$, large $H$, and moderate $W$ so that $P_{b}^{1 / W}$ is not too close to unity.

It is also reported in [44] that the gain increases as the blocking probability decreases, but this effect is small for small values of $P_{b}$. Also, as $W$ increases, $G$ also increases until it peaks around $W \approx 10$ (for $q \approx 0.5$ ), and the maximum gain is close to $H / 2$. After peaking, $G$ decreases, but very slowly. Generally, it is found that for a moderate to large number of wavelengths, the benefits of wavelength conversion increase with the length of the connection, and decrease (slightly) with an increase in the number of wavelengths. While this was a simple analysis to study the effects of hop length, a more rigorous treatment incorporating the load dependencies on successive links in a path is also presented in [44].

\section{B. Related Work on Gain Characterization}

In this section, we present other significant works which characterize the gain available from networks with wavelength converters. 
1) Bounds on RWA Algorithms with and Without Wavelength Converters [36]: Upper bounds on the carried traffic (or equivalently, lower bounds on the blocking probability) in a wavelength-routed WDM network are derived in [36]. The bounds are shown to be achievable asymptotically by a fixed RWA algorithm using a large number of wavelengths. The wavelength reuse factor-which is defined as the maximum offered traffic per wavelength for which the blocking probability can be made arbitrarily small by using a sufficiently large number of wavelengths-is found to increase by using wavelength converters in large networks. Simulations show that wavelength converters offer a $10-40 \%$ increase in the amount of reuse available in the authors' sampling of 14 networks ranging from 16 to 1000 nodes when the number of wavelengths available is small (10 or 32).

2) Multifiber Networks [30]: The benefits of wavelength conversion in a network with multiple fiber links are studied in [30], by extending the analysis presented in [44] to multifiber networks. Multifiber links are found to reduce the gain obtained due to wavelength conversion, and the number of fibers is found to be more important than the number of wavelengths for a network. It is concluded that a mesh network enjoys a higher utilization gain with wavelength conversion for the same traffic demand than a ring or a fully connected network.

3) Limited-Range Wavelength Conversion [28]: The effects of limited-range wavelength conversion (see Section IIIA) on the performance gains achievable in a network are considered in [28]. The model used in this work captures the functionality of certain all-optical wavelength converters (e.g., those based on four-wave mixing) whose conversion efficiency drops with increasing range. The analytical model follows from [44], but employs both link-load-independence and wavelength-independence assumptions. The results obtained indicate that a significant improvement in the blocking performance of the network is obtained when limitedrange wavelength converters with as little as one-quarter of the full range are used. Moreover, converters with just half of the full conversion range deliver almost all of the performance improvement offered by an ideal full range converter.

4) Minimal Wavelength Conversion in WDM Rings [45]: In addition to reducing the overall blocking probability, wavelength converters can improve the fairness performance by allowing many long-distance lightpaths, which would have been otherwise blocked, to be established [24]. In [45], the authors define the unfairness factor as the ratio of the average blocking on the longest path to the blocking on the shortest path. The fairness ratio is then defined as the ratio of the unfairness factor without wavelength conversion to that with wavelength conversion. Simulation studies in a 195-node network of 15 interconnected WDM rings with 13 nodes each show significant increase in fairness ratio, of approximately 10000 , for 32 wavelengths. Similar trends have also been observed in smaller rings. Moreover, for large interconnected rings, this improvement can be achieved with wavelength conversion in just $10-20 \%$ of the nodes.

\section{SUMMARY}

In this study, we examined the various facets of the wavelength-conversion technology from its realization using current $\mathrm{OE}$ devices, to its incorporation in a wavelengthrouted network design, to its effect on efficient routing and management algorithms, to a measurement of its potential benefits under various network conditions. While understanding of the technology has improved during the past few years, several issues still remain unresolved, especially in the context of efficient design mechanisms and routing protocols. Additional efforts in this area are needed to further the performance of such networks using intelligent routing and design methods.

\section{REFERENCES}

[1] C. A. Brackett, "Dense wavelength division multiplexing networks: Principles and applications," IEEE J. Select. Areas Commun., vol. 8, pp. 948-964, Aug. 1990.

[2] B. Mukherjee, Optical Communication Networks. New York: McGraw-Hill, 1997.

[3] C. A. Brackett et al., "A scalable multiwavelength multihop optical network: A proposal for research on all-optical networks," J. Lightwave Technol., vol. 11, pp. 736-753, May/June 1993.

[4] B. S. Glance, J. M. Wiesenfeld, U. Koren, and R. W. Wilson, "New advances on optical components needed for FDM optical networks," $J$. Lightwave Technol., vol. 11, pp. 882-890, May/June 1993.

[5] T. Durhuus et al., "All-optical wavelength conversion by semiconductor optical amplifiers," J. Lightwave Technol., vol. 14, pp. 942-954, June 1996.

[6] P. Rigole, S. Nilsson, E. Berglind, and D. J. Blumenthal, et al., "State of the art: Widely tunable lasers. (In-plane semiconductor lasers: From ultraviolet to midinfrared," in Proc. SPIE, vol. 3001, San Jose, CA, USA, Feb. 1997, pp. 382-393.

[7] J. M. Wiesenfeld, "Wavelength conversion techniques," in Proc. OFC '96, San Jose, CA, Feb. 1996, pp. 71-72, paper TuP 1.

[8] M. Fujiwara et al., "A coherent photonic wavelength-division switching system for broadband networks," in Proc. ECOC '88, Brighton, U.K., Sept. 1988, pp. 139-142.

[9] D. J. G. Mestdagh, Fundamentals of Multiaccess Optical Fiber Networks. Norwood, MA: Artech House, 1995.

[10] S. J. B. Yoo, "Wavelength conversion technologies for WDM network applications," J. Lightwave Technol., vol. 14, pp. 955-966, June 1996.

[11] R. W. Tkach et al., "Four-photon mixing and high-speed WDM systems," J. Lightwave Technol., vol. 13, pp. 841-849, May 1995.

[12] R. Schnabel et al., "Polarization insensitive frequency conversion of a 10-channel OFDM signal using four-wave mixing in a semiconductor laser amplifier," IEEE Photon. Technol. Lett., vol. 6, pp. 56-58, Jan. 1994.

[13] R. Ludwig and G. Raybon, "BER measurements of frequency converted signals using four-wave mixing in a semiconductor laser amplifier at 1 , 2.5, 5, and 10 Gbit/s," Electron. Lett.., vol. 30, pp. 338-339, Jan. 1994.

[14] J. Zhou et al., "Four-wave mixing wavelength conversion efficiency in semiconductor traveling-wave amplifiers measured to 65-nm of wavelength shift," IEEE Photon. Technol. Lett., vol. 6, pp. 984-987, Aug. 1994.

[15] N. Antoniades, K. Bala, S. J. B. Yoo, and G. Ellinas, "A parametric wavelength interchanging cross-connect (WIXC) architecture," IEEE Photon. Technol. Lett., vol. 8, pp. 1382-1384, Oct. 1996.

[16] J. P. R. Lacey, G. J. Pendock, and R. S. Tucker, "Gigabit-per-second alloptical 1300-nm to 1550 -nm wavelength conversion using cross-phase modulation in a semiconductor optical amplifier," in Proc. OFC '96, San Jose, CA, Feb. 1996, vol. 2, pp. 125-126.

[17] M. Eiselt, W. Pieper, and H. G. Weber, "Decision gate for all-optical retiming using a semiconductor laser amplifier in a loop mirror configuration," Electron. Lett.., vol. 29, pp. 107-109, Jan. 1993.

[18] T. Durhuus et al., "All optical wavelength conversion by SOA's in a Mach-Zender configuration," IEEE Photon. Technol. Lett., vol. 6, pp. 53-55, Jan. 1994.

[19] B. Mikkelsen et al., "Polarization insensitive wavelength conversion of 10 Gbit/s signals with SOA's in a Michelson interferometer," Electron. Lett.., vol. 30, pp. 260-261, Feb. 1994. 
[20] _ "All-optical noise reduction capability of interferometric wavelength converters," Electron. Lett.., vol. 32, pp. 566-567, Mar. 1996.

[21] H. Yasaka et al., "Finely tunable $10-\mathrm{Gb} / \mathrm{s}$ signal wavelength conversion from 1530- to 1560-nm region using a super structure grating distributed Bragg reflector laser," IEEE Photon. Technol. Lett., vol. 8, pp. 764-766, June 1996.

[22] B. Mikkelsen et al., "Wavelength conversion devices," in Proc. OFC '96, San Jose, CA, vol. 2, 1996, pp. 121-122.

[23] R. Sabella and E. Iannone, "Wavelength conversion in optical transport networks," Fiber Integr. Opt., vol. 15, no. 3, pp. 167-191, 1996.

[24] K.-C. Lee and V. O. K. Li, "A wavelength-convertible optical network," J. Lightwave Technol., vol. 11, pp. 962-970, May/June 1993.

[25] J. Iness, "Efficient use of optical components in WDM-based optical networks," Ph.D. dissertation, Univ. California, Davis, 1997.

[26] M. Kovačević, and A. S. Acampora, "Electronic wavelength translation in optical networks," J. Lightwave Technol., vol. 14, pp. 1161-1169, June 1996.

[27] S. Subramaniam, M. Azizoḡlu, and A. K. Somani, "All-optical networks with sparse wavelength conversion," IEEE/ACM Trans. Networking, vol. 4, pp. 544-557, Aug. 1996.

[28] J. Yates, J. Lacey, D. Everitt, and M. Summerfield, "Limited-range wavelength translation in all-optical networks," in Proc. INFOCOM '96, 1996, San Francisco, CA, Mar. 1996, pp. 954-961.

[29] R. Ramaswami and G. H. Sasaki, "Multiwavelength optical networks with limited wavelength conversion," in Proc., INFOCOM '97, Kobe, Japan, Apr. 1997, pp. 490-499.

[30] G. Jeong and E. Ayanoglu, "Comparison of wavelength-interchanging and wavelength-selective cross-connects in multiwavelength all-optical networks," in Proc. INFOCOM '96, San Francisco, CA, Mar. 1996, pp. $156-163$.

[31] O. Gerstel, R. Ramaswami, and G. Sasaki, "Fault tolerant multiwavelength optical rings with limited wavelength conversion," in Proc. INFOCOM '97, Kobe, Japan, Apr. 1997, pp. 508-516.

[32] K. Bala, T. E. Stern, and K. Bala, "Algorithms for routing in a linear lightwave network," in Proc. INFOCOM '91, Bal Harbour, FL, Apr. 1991, pp. 1-9.

[33] D. Banerjee and B. Mukherjee, "A practical approach for routing and wavelength assignment in large wavelength-routed optical networks," IEEE J. Select. Areas Commun., vol. 14, pp. 903-908, June 1996.

[34] I. Chlamtac, A. Ganz, and G. Karmi, "Lightpath communications: An approach to high-bandwidth optical WAN's," IEEE Trans. Commun., vol. 40, pp. 1171-1182, July 1992.

[35] I. Chlamtac, A. Faragó, and T. Zhang, "Lightpath (wavelength) routing in large WDM networks," IEEE J. Select. Areas Commun., vol. 14, pp. 909-913, June 1996.

[36] R. Ramaswami and K. N. Sivarajan, "Routing and wavelength assignment in all-optical networks," IEEE/ACM Trans. Networking, vol. 3, pp. 489-500, Oct. 1995.
[37] C. Chen and S. Banerjee, "A new model for optimal routing and wavelength assignment in wavelength division multiplexed optical networks," in Proc. INFOCOM '96, San Francisco, CA, Mar. 1996, pp. 164-171.

[38] R. Ramaswami, "Optical network architectures," in Proc. OFC '96, San Jose, CA, Feb. 1996, vol. 2, p. 223.

[39] N. Wauters, W. V. Parys, B. V. Caenegem, and P. Demeester, "Reduction of wavelength blocking through partitioning with wavelength converters," in Proc. OFC '97, 1997, vol. 6, pp. 122-123.

[40] M. Kovačević and A. S. Acampora, "Benefits of wavelength translation in all-optical clear-channel networks," IEEE J. Select. Areas Commun., vol. 14, pp. 868-880, June 1996.

[41] F. P. Kelly, "Blocking probabilities in large circuit switched networks," Adv. Appl. Prob., vol. 18, pp. 473-505, 1986.

[42] S. Chung, A. Kashper, and K. W. Ross, "Computing approximate blocking probabilities for large loss networks with state-dependent routing," IEEE/ACM Trans. Networking, vol. 1, no. 1, pp. 105-115, 1993.

[43] A. Birman, "Computing approximate blocking probabilities for a class of all-optical networks," IEEE J. Select. Areas Commun., vol. 14, pp. 852-857, June 1996.

[44] R. A. Barry and P. A. Humblet, "Models of blocking probability in all-optical networks with and without wavelength changers," IEEE $J$. Select. Areas Commun., vol. 14, pp. 858-867, June 1996.

[45] K. Bala, E. Bouillet, and G. Elinas, "The benefits of 'minimal' wavelength interchange in WDM rings,' in Proc. OFC '97, Dallas, TX, Feb. 1997, vol. 6, pp. 120-121.

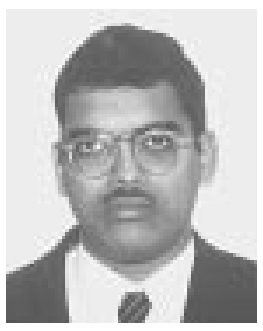

Byrav Ramamurthy (S'97) received the B.Tech. degree in computer science and engineering from the Indian Institute of Technology, Madras, in 1993 He received his M.S. and Ph.D. degrees in computer science from the University of California (UC), Davis, in 1995 and 1998, respectively.

Since August 1998, he has been an Assistant Professor in the Department of Computer Science and Engineering at the University of Nebraska-Lincoln. His research interests include high-speed computer networks, distributed systems, and telecommunications.

Dr. Ramamurthy received the Indian National Talent Search scholarship and was a fellow of the Professors for the Future program at UC Davis.

Biswanath Mukherjee (S'82-M'87), for a photograph and biography, see this issue, p. 1050 